\title{
SYSTEMIC LUPUS ERYTHEMATOSUS WITH VALVE IMPAIRMENT - LIBMAN-SACKS ENDOCARDITIS: CASE REPORT
}

Gustavo Lawin ${ }^{1, \star}$, Milene Tieno Sakata Vasconcellos ${ }^{1}$, Rodrigo Maldonado Franco ${ }^{1}$, Bruno Carnevalli ${ }^{1}$, Meline Angelica Cunha Rotter Ferreira ${ }^{1}$, Anna Herminia Castro Gomes de Amorim¹․ Cecilia Resende Brunow Bazzo ${ }^{1}$, Luiz Eduardo de Paula ${ }^{1}$, Neide Tomimura Costa ${ }^{1}$, Priscila Beatriz de Souza Medeiros ${ }^{1}$

1.Universidade Estadual de Londrina, Londrina (PR), Brazil.

${ }^{\star}$ Corresponding author: gustavolawin@hotmail.com

\section{BACKGROUND}

The Libman-Sacks endocarditis (LSE) is characterized by the presence of vegetation in the heart valves not secondary to the action of infectious agents. It is a pathology associated with diseases such as antiphospholipid syndrome (APS) and/or systemic lupus erythematosus (SLE). The mitral valve is the most frequently affected part. The LSE is often asymptomatic, but fragmentation of vegetation with systemic embolism can occur.

\section{CASE REPORT}

Woman, 23 years old, former addict (marijuana, cocaine, and tobacco), with a 3-year history: arthralgia of hands and wrists, cicatricial alopecia, malar rash, oral ulcers, fatigue, and unfocused fever. Under investigation, the hypothesis of SLE was confirmed, associated with lupus nephritis and APS without possibility of biopsy at that time. After a methylprednisolone pulse, the patient was discharged with hydroxychloroquine $400 \mathrm{mg} /$ day, prednisone $1 \mathrm{mg} / \mathrm{kg} / \mathrm{day}$, and acetylsalicylic acid $100 \mathrm{mg} / \mathrm{day}$. She remained stable, but with difficulty adhering to the treatment, abandoned within a year. Then, she sought care with a condition of anasarca and oliguria, and a renal biopsy was performed with the following report: global (6/14) and partial (8/14) glomerular sclerosis with synechiae and fibrocellular crescent (1/14); multifocal tubular atrophy with moderate interstitial fibrosis; moderate fibrous hyperplasia of the arterial intima. The patient needed to start and maintain hemodialysis. Due to disease activity, a new pulse therapy was performed. She was discharged and exams were requested for her follow-up in early return. Among the results, there was a transthoracic echocardiogram with this unexpected report: LV concentric hypertrophy: moderate degree; LVEF 40\%/PASP 70 mmHg; diffuse LV impairment: moderate degree; mitral regurgitation: major degree; tricuspid insufficiency: major degree; pericardial effusion: moderate degree; thickening of the posterior mitral valve leaflet — infectious process? The hypothesis of LSE was considered and evaluated by the cardiology staff, which ruled out other causes and contraindicated surgery at that time. The patient was clinically well and, as it is a high-risk surgery, it was decided to start cyclophosphamide, six monthly cycles, with response reassessment in six months.

\section{CONCLUSION}

As seen, he patient's cardiac condition was asymptomatic, and LSE was an imaging finding in her routine in our clinic. As it is often a silent disease, manifesting itself late with thromboembolic events, we must always pay close attention to the patient, even without complaining during the query. Thus, further complications can be avoided by having early diagnoses and proper treatments.

\section{KEYWORDS}

Lupus, Valve, Libman, Endocarditis. 